\title{
The analysis of prognosis factor in patients with non-small cell lung cancer receiving pneumonectomy
}

\author{
Guige Wang, Lei Liu, Jiaqi Zhang, Shanqing Li \\ Department of Thoracic Surgery, Peking Union Medical College Hospital, Chinese Academy of Medical Sciences and Peking Union Medical \\ College, Dongcheng District, Beijing 100730, China \\ Contributions: (I) Conception and design: S Li; (II) Administrative support: S Li; (III) Provision of study materials or patients: S Li; (IV) Collection \\ and assembly of data: G Wang, J Zhang, L Liu; (V) Data analysis and interpretation: G Wang, L Liu; (VI) Manuscript writing: All authors; (VII) \\ Final approval of manuscript: All authors. \\ Correspondence to: Shanqing Li. Department of Thoracic Surgery, Peking Union Medical College Hospital, Chinese Academy of Medical Sciences \\ and Peking Union Medical College, Dongcheng District, Beijing 100730, China. Email: 839791089@qq.com.
}

Background: Pneumonectomy is a procedure that possesses several side effects, but is sometimes necessary in the management of non-small cell lung cancer (NSCLC). The benefits of pneumonectomy have yet to be clearly outlined.

Methods: Data of 100 cases were extracted from the medical records of patients that underwent a pneumonectomy for NSCLC from January 2007 to December 2016. Primary outcomes were 5-year overall survival (OS) and 30-day mortality. Statistical comparisons were performed using the Chi-Square test. Kaplan-Meier curves were utilized to evaluate the 5-year OS which were compared using the log-rank test. Multivariable analysis of survival data was done using risk proportional model.

Results: The 5-year OS of NSCLC after pneumonectomy is $32.3 \%$. Squamous cell carcinomas had a better prognosis than adenocarcinomas $(\mathrm{P}=0.039)$. Patients with higher $\mathrm{N}$ stage had a worse prognosis. Among patients undergoing pneumonectomy with N2 lymphatic metastasis, those who also underwent neoadjuvant therapy achieved a better 5 -year OS $(\mathrm{P}=0.042)$. The 30 -day mortality was $4.0 \%$.

Conclusions: Pneumonectomy sometimes is inevitable and necessary in certain subtypes of NSCLC with acceptable perioperative mortality and long-term survival. For patients with NSCLC undergoing pneumonectomy, pathological diagnosis and nodal stage were independent predictors of OS. When pneumonectomy was done in patients with NSCLC and N2 lymphatic metastasis, a better long-term OS could be achieved amongst patients receiving neoadjuvant therapy compared to those without neoadjuvant therapy.

Keywords: Neoadjuvant therapy; non-small cell lung cancer (NSCLC); overall survival (OS); pneumonectomy

Submitted Oct 08, 2019. Accepted for publication Jan 18, 2020.

doi: $10.21037 /$ jtd.2020.02.33

View this article at: http://dx.doi.org/10.21037/jtd.2020.02.33

\section{Introduction}

Lung cancer is a malignant tumor that possesses high rates of morbidity and mortality, with more than 200,000 patients diagnosed with lung cancer in the United States each year (1). Over $80 \%$ of all lung cancer is of the nonsmall cell lung cancer (NSCLC) subtype. Surgery plays a dominant role in the management of stage I, II and some
IIIA NSCLC, with lobectomy being the golden standard. However, in certain circumstances such as a centrally located tumor and great vessel invasion, a pneumonectomy would be necessary to achieve radical resection of the lesion. The surgery extent of pneumonectomy for NSCLC include the anatomic resection of hemi-pulmonary tissue and pulmonary artery, vein and main bronchus, as well as systematic lymph node dissection. Dr. Graham reported 
the first pneumonectomy for lung cancer in the fournal of American Medical Association (JAMA) in 1933 (2). Since then, lung cancer treatment has been optimized to a combination of surgery, chemotherapy and radiotherapy. However, pneumonectomy could lead to severe damage of pulmonary function, hemodynamic change and further decreased cardiac function $(3,4)$. Therefore, careful consideration should be taken for patients that are candidates for pneumonectomies.

\section{Methods}

\section{Patients}

A total of 132 consecutive patients who presented to Peking Union Medical College Hospital from January 2007 to December 2016 and received pneumonectomy were included in this study. The exclusion criteria were as follows: (I) benign disease, including bronchiectasis and a destroyed lung; (II) lung malignant disease that were not NSCLC; (III) undefined cancers; (IV) distant metastasis; (V) metastatic lung cancer. According to the exclusion criteria, 8 patients undergone pneumonectomy with benign diseases, 16 patients had small cell lung cancer (SCLC), 3 patients had neuroendocrine carcinoma (without a more concrete pathological type), 2 patients had residual lung resection due to recurrence, 3 patients had distant metastasis and with uncontrollable massive hemoptysis. All of these 32 cases were excluded and 100 patients were finally included in the study.

\section{The indications and procedure of pneumonectomy}

Pneumonectomy is inevitable if there is direct tumor invasion to the main pulmonary artery or interlobar pulmonary artery, or when the lymph nodes surround the interlobar artery or the root of the pulmonary lobar/ segmental artery, or when the lesions invade and cross the adjacent lobes, or when the lesions in the bronchial cavity exceed the limits of lobectomy and sleeve lobectomy. Pneumonectomies were started with a posterolateral incision, followed by a comprehensive exploratory thoracotomy to ensure that the tumor could be resected completely and that the goal of complete resection was achievable. It was essential to determine if the pulmonary artery could be released safely after preliminary dissociation of pulmonary hilum. If a complete resection was not possible without opening the pericardium, the exposure of intrapericardial pulmonary artery and vein is necessary.
A lymphadenectomy of the mediastinal lymph nodes was an integral part of the procedure. Bronchial stumps were sometimes covered with an intercostal muscle flap.

\section{Survival analysis of 100 patients}

The age, sex, side, size, pathological type, neoadjuvant therapy, stage and whether adjuvant therapy was performed were collected with all 100 patients undergoing regular follow-up. All of the patients receiving neoadjuvant therapy were diagnosed histopathologically through biopsies and the diagnosis of lymphatic metastasis was confirmed by bronchoscopic biopsy or positron emission tomography (PET) [the standardized uptake value (SUV) threshold of 2.5 is generally used to designate a scan as positive] when pathological proof was not accessible. The lung cancer stage was determined in accordance to the eighth edition of Union for International Cancer Control (UICC). The data were collected and further analyzed to evaluate prognosis. The research was approved by the institutional review board and written consent was obtained from each patient.

\section{Survival analysis of patients with N2 lymphatic metastasis}

Patients undergoing pneumonectomy with N2 lymphatic metastasis were filtered to analyze the effect of neoadjuvant therapy to survival.

\section{Statistical analysis}

Statistical analysis was performed using EXCEL and SPSS 24.0. Survival was calculated using the Kaplan-Meier method and was statistically evaluated by the log-rank test. The COX hazard proportion model was used to determine survival through multivariate analysis. A $\mathrm{P}$ value of less than 0.05 was considered significant.

\section{Results}

\section{Descriptive analysis}

Based on the exclusion criteria, 100 patients who received pneumonectomy were analyzed (Table 1), including 89 males $(89.0 \%)$ and 11 females (11.0\%), with the average age of 57.14 (ranged from 26 to 77 ) years old. Squamous cell carcinoma was more common than adenocarcinoma, while left-sided pneumonectomies' were more common than right-sided pneumonectomies. Mediastinal node metastasis 
Table 1 Clinicopathological characteristics of patients under pneumonectomy, with and without neoadjuvant therapy

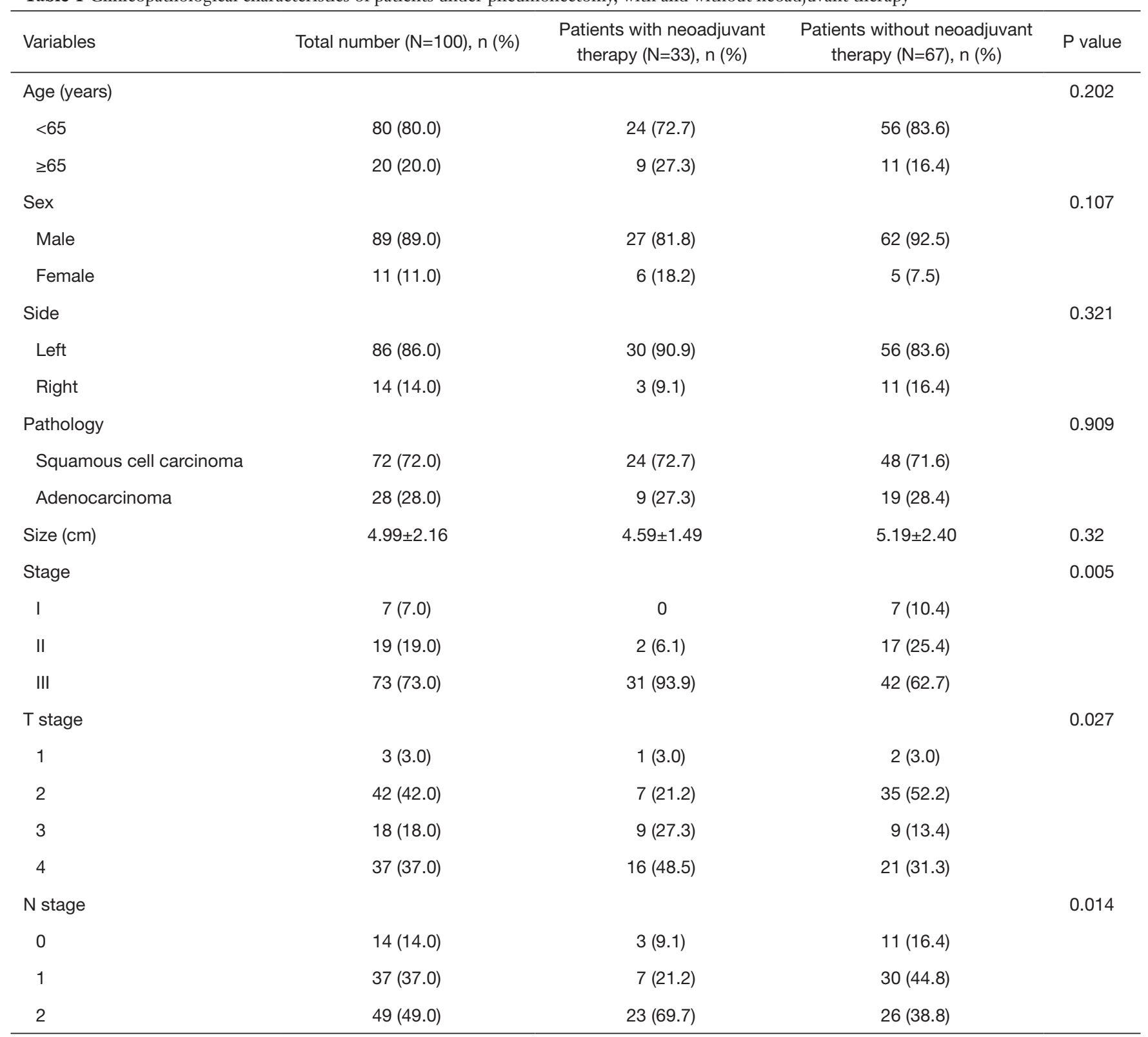

was confirmed by histopathology in 29 patients $(87.9 \%)$ and by PET in 4 patients $(12.1 \%$ ) (4 of these patients did not receive histopathological diagnosis given their high risk of vascular injury) prior to neoadjuvant therapy.

\section{Survival analysis of 100 patients (Table 2)}

All 100 patients underwent an anatomic pneumonectomy and systematic lymphadenectomy, with no extended resection. The median survival time was 36 months.
One patient received an $\mathrm{R} 1$ resection and received postoperative chemoradiotherapy and a follow-up period of 83 months, and this patient was still alive. The 5 -year overall survival (OS) rate was $32.3 \%$. The 5 -year OS time was not statistically different between patients elder than 65 years old and patients younger than 65 years old $(\mathrm{P}=0.231)$. Likewise, no statistically significant differences were found for 5 -year OS amongst different genders, tumor sizes, tumor sides and $\mathrm{T}$ stage. Squamous cell carcinoma possessed a better 5 -year OS than adenocarcinoma $(36.5 \%$ 
Table 2 Univariate analysis of 5-year OS

\begin{tabular}{llll}
\hline Variables & HR & 95\% Cl & P value \\
\hline Age (years) & 1.431 & 0.790 to 2.590 & 0.231 \\
Sex & 0.715 & 0.339 to 1.506 & 0.372 \\
Side & 0.863 & 0.439 to 1.696 & 0.666 \\
Pathology & 0.586 & 0.350 to 0.982 & 0.039 \\
Size (cm) & 1.023 & 0.627 to 1.667 & 0.928 \\
Stage & 1.899 & 1.209 to 2.983 & 0.017 \\
T stage & 1.005 & 0.780 to 1.295 & 0.894 \\
N stage & 2.125 & 1.457 to 3.098 & 0.000 \\
Neoadjuvant therapy & 0.813 & 0.476 to 1.388 & 0.444 \\
Adjuvant therapy & 1.206 & 0.656 to 2.217 & 0.543 \\
\hline
\end{tabular}

OS, overall survival.

Table 3 Multivariate analysis of 5-year OS

\begin{tabular}{lccc}
\hline Variables & HR & $95 \% \mathrm{Cl}$ & $\mathrm{P}$ value \\
\hline Pathology & 0.566 & 0.331 to 0.968 & 0.038 \\
Stage & 1.080 & 0.592 to 1.970 & 0.803 \\
N stage & 2.148 & 1.307 to 3.532 & 0.003 \\
\hline
\end{tabular}

OS, overall survival.

vs. $20.4 \%, \mathrm{P}=0.039)$. The 5 -year OS rates of stage I, II and III were $71.4 \%, 32.4 \%$ and $28.7 \%(\mathrm{P}=0.017)$, respectively. The OS rates of lymph node stage 0,1 and 2 were $63.5 \%$, $35.3 \%$ and $19.6 \%(\mathrm{P}=0.000)$, respectively. There was no difference in 5 -year survival rates between patients with and without neoadjuvant therapy ( $39.8 \%$ vs. $29.2 \%, \mathrm{P}=0.444$ ), so was adjuvant therapy $(\mathrm{P}=0.543)$. In the multivariable analysis, pathology and $\mathrm{N}$ stage were independent predictors of 5-year OS (Table 3). The 30-day mortality was $4.0 \%$, resulting from bronchopleural fistula, acute respiratory distress and cerebral infarction.

\section{The survival analysis of patients with N2 lymphatic metastasis}

Of the 49 patients, 36 patients reached the endpoint of the study. Thirteen patients were alive, including 3 patients living longer than 5 years. The median follow-up time of N2 positive patients was 15 months. The median survival time of N2 patients was 21 months. Twenty-three patients (46.9\%) underwent neoadjuvant therapy, including 22 patients who received neoadjuvant chemotherapy, 1 patient who received neoadjuvant chemoradiotherapy and 42 patients $(85.7 \%)$ who received adjuvant therapy. These 23 patients who received neoadjuvant therapy had a better 5 -year OS than those without neoadjuvant therapy (29.3\% vs. $11.1 \%, \mathrm{P}=0.042$ ) (Table 4, Figure 1). Among those 23 patients with N2 lymphatic metastasis who received neoadjuvant therapy, only 6 patients $(26.1 \%)$ were observed to have lymph node downstaging but without 5 -year survival benefit $(\mathrm{P}=0.473)$. Among the 26 patients without neoadjuvant therapy, 22 patients $(84.6 \%)$ received adjuvant therapy and the other 4 patients didn't receive either neoadjuvant or adjuvant therapy. One patient died because of bronchopleural fistula, 1 patient died of acute respiratory distress, the other 2 patients were elder than 70 years old and was not able to tolerate adjuvant therapy. Only 1 of these 4 patients survived over 6 months.

\section{Discussion}

Pneumonectomy is a rare procedure in pulmonary surgery 
Table 4 Five-year survival for patients with N2 lymphatic metastasis who underwent pneumonectomy for NSCLC, with and without neoadjuvant therapy

\begin{tabular}{lccccc}
\hline \multirow{2}{*}{ Treatment } & \multicolumn{2}{c}{ Total patients } & & \multicolumn{2}{c}{ 5-year survival } \\
\cline { 2 - 3 } \cline { 5 - 6 } & Number & Percentage & & Alive number & Percentage \\
\hline With neoadjuvant therapy & 23 & 46.94 & 4 & 29.30 \\
Without neoadjuvant therapy & 26 & 53.06 & 1 & 11.10 & 0.042 \\
Total & 49 & 100.00 & 5 & 19.60 \\
\hline
\end{tabular}

NSCLC, non-small cell lung cancer.

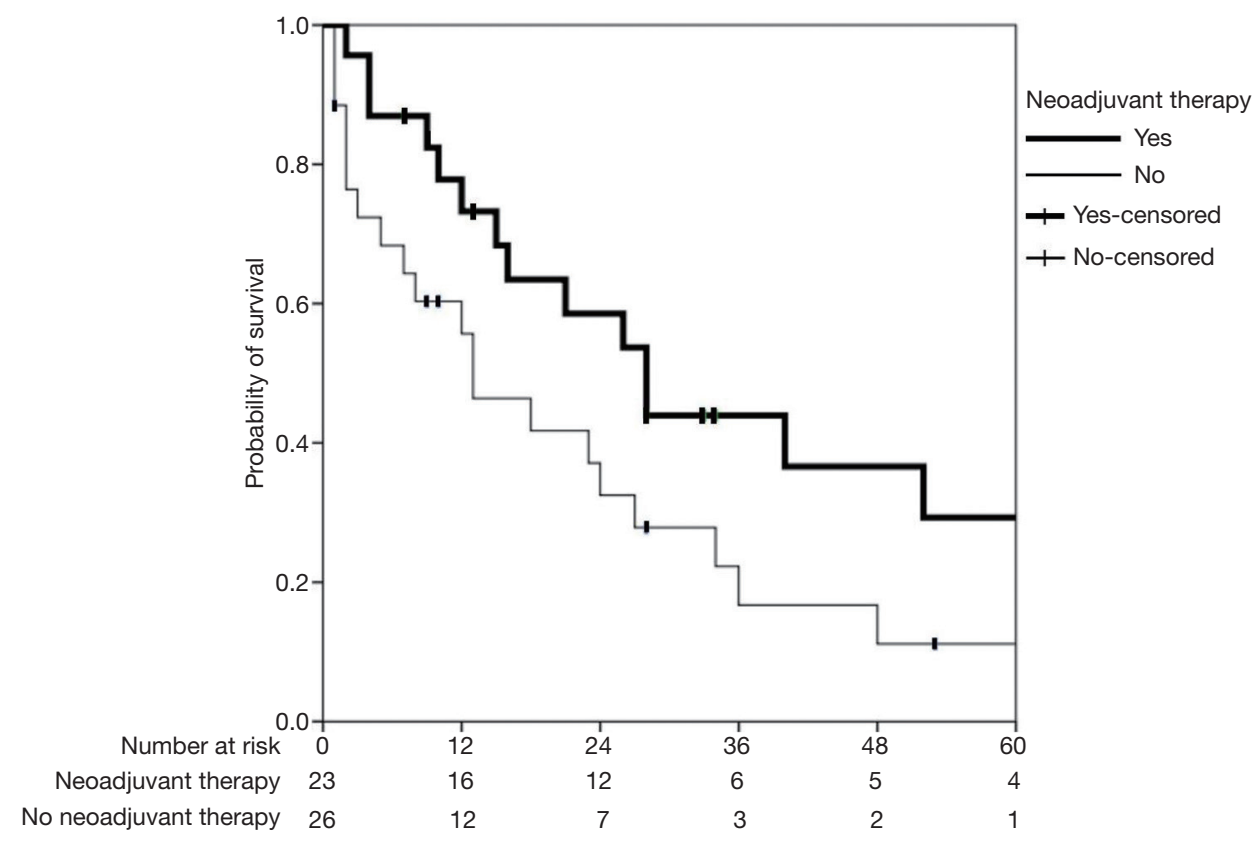

Figure 1 Kaplan-Meier curve in patients receiving pneumonectomy with N2 lymphatic metastasis. Patients receiving neoadjuvant therapy had a better 5 -year OS than those without neoadjuvant therapy (29.3\% vs. $11.1 \%, \mathrm{P}=0.042)$. OS, overall survival.

with high perioperative morbidities and post-operative mortality, such as acute loss of pulmonary function and increasing respiratory and circulatory load, and even death. Lobectomy with or without bronchoplasty or arterioplasty, or sleeve lobectomy is the preferred choice in contrast to pneumonectomy in central or locally advanced lung cancer, such as tumor invasion of the main pulmonary artery or interlobar pulmonary artery, or lymph node invasion of the interlobar artery or the root of the pulmonary lobar/ segmental artery, or invasion of lesions to adjacent lobes. However, the procedure of pneumonectomy is often the most suitable mode of surgery in cases where tumor or lymph node invasion exceed the limits of lobectomy or sleeve lobectomy. From January 2007 to December 2016, we performed 132 pneumonectomies accounting for $1.6 \%$ of the total number of operations, 111 sleeve resections accounting for $1.4 \%$ of operations and 148 lobectomies with plasty of bronchus or artery accounting for $1.80 \%$ of operations. The percentage of both pneumonectomy and sleeve resections decreased gradually with increasing popularity of pulmonary resections, with over 2,000 pulmonary resections performed by our center in 2019 .

Perioperative mortality of pneumonectomy in recent years are on decline. Licker et al. reported a 30-day mortality of $9.3 \%$ (5); Shapiro et al. reported a perioperative mortality of $5.6 \%$ (6); and Ludwig et al. reported a perioperative mortality of $4.6 \%$ (7). Qadri et al. reported that intensive post-operative management could reduce the 
perioperative mortality to $3.3 \%$ (8). In our institute, 30-day mortality of 100 patients under pneumonectomy was $4.0 \%$. With acceptable perioperative mortality and long-term OS, pneumonectomy still plays an important role in the treatment of NSCLC (9-11).

Age is an important predictor for long-term survival among NSCLC patient who have received pneumonectomy. The long-term survival shortens with increasing age, especially in stage I NSCLC (12). The cut-off age of 70 years old was advised most often to evaluate the prognosis of NSCLC under pneumonectomy $(13,14)$. In our institute, there were only 8 patients $(8.0 \%)$ elder than 70 years old and no perioperative mortality and long-term survival differences were observed. When 65 years were adopted as cut off value, the number of elderly patients was 20 (20.0\%). According to the Kaplan-Meier curve, elder patients tended to show worse prognosis but without statistical difference $(\mathrm{P}=0.231)$. We failed to prove whether the age was a predictor of poor prognosis, possibly due to the small samples of elderly patients in our study. Although the cut-off value of 65 years increased the numbers of elderly patients, the cut-off value predicting prognosis might not be 65 years. Therefore, patients should not be denied pneumonectomy solely due to elder age and should be carefully considered in cases where a pneumonectomy was indicated.

Tumor side was previously found to have an impact on perioperative mortality (15). In our institute, 86 patients $(86.0 \%)$ who received a left pneumonectomy had a 90 -day mortality of $3.5 \%$, compared to those who received a right pneumonectomy had a 90 -day mortality of $21.4 \%$. The 5 -year OS were $37.7 \%$ vs. $30.1 \%(\mathrm{P}=0.666)$, respectively. Likewise, Fernandez et al. reviewed 9,746 patients with NSCLC who had undergone pneumonectomy (left accounted for $56 \%$ ) and found that the 30-day and 90 mortality was $8 \%$ and $16 \%$ for right pneumonectomy and $4 \%$ and $9 \%$ for left $(\mathrm{P}<0.001)$. This article proved the lesion side was a predictor of short-term mortality, but without impact on long-term survival (16). We failed to demonstrate any significant differences of short-term mortality and long-term survival caused by lesion side, possibly due to the small samples of those who received right pneumonectomy in our study.

Kaplan-Meier curve showed that squamous cell carcinoma had better 5 -year OS than adenocarcinoma (36.5\% vs. $20.4 \%, \mathrm{P}=0.039$ ). Squamous cell carcinoma was a predictor of better long-term prognosis. Gu et al. reported that patients with adenocarcinoma who underwent pneumonectomy had a worse prognosis compared to those who had squamous cell carcinoma, but without statistical significance $(\mathrm{P}=0.172)$ (17).

Thirty-three patients who were enrolled in our institute underwent neoadjuvant therapy. The Kaplan-Meier curve showed that patients with neoadjuvant therapy demonstrated no significant 5 -year OS benefit compared to those without neoadjuvant therapy ( $39.8 \%$ vs. $29.2 \%, \mathrm{P}=0.444)$. Similarly, $\mathrm{Gu} e t \mathrm{al}$. found that neoadjuvant therapy could not improve prognosis (17). Our study shows that patients who received adjuvant therapy had no 5 -year OS benefit compared to those without adjuvant therapy $(\mathrm{P}=0.543)$. Lymphatic metastasis remarkably affected prognosis and a higher $\mathrm{N}$ stage and tumor stage led to worse prognosis.

In our institution, we had a total of 45 patients with $\mathrm{T} 1$ and $\mathrm{T} 2$ stage tumor who accounted for $45 \%$ of patients receiving pneumonectomy. After analysis, we found that most of the patients had T2 stage (93.3\%), N1-2 lymph node metastasis $(84.4 \%)$ and squamous cell carcinoma (73.3\%). In adenocarcinoma, 9 patients $(75.0 \%)$ had IIIA stage and 3 patients $(25.0 \%)$ had IIB stage. However, in patients squamous cell carcinoma, 7 patients $(21.2 \%)$ had IA or IB stages, with the other 26 patients $(78.8 \%)$ had IIB or IIIA stages. In 45 patients with T1 and T2, these 7 patients with squamous cell carcinoma accounted for all of cohort of stage I patients who received pneumonectomies, which was performed because of the massive hemorrhage attributed to the central characteristics of squamous cell carcinoma and severe inflammatory adhesion. Univariable analysis revealed that pathological subtype, tumor stage and $\mathrm{N}$ stage were all predictors of the 5-year OS. But in multivariable analysis, pathological subtype and $\mathrm{N}$ stage were predictors of 5-year OS (Table 3).

Forty-nine patients undergoing pneumonectomy with $\mathrm{N} 2$ lymphatic metastasis were divided into two groups. The neoadjuvant therapy group included 23 patients and the non-neoadjuvant therapy group included 26 patients. We found that patients who received neoadjuvant therapy had a better 5-year OS compared to those without neoadjuvant therapy (29.3\% vs. $11.1 \%, \mathrm{P}=0.042$ ) (Table 2). Lee et al. (14), Marulli et al. (18) all also demonstrated that patients with stage III NSCLC and N2 lymphatic metastasis could benefit from neoadjuvant therapy as it was shown to reduce morbidities and mortality while improving long-term survival, especially for those who were responsive to neoadjuvant therapy (18). In another retrospective research enrolling 
240 NSCLC patients who underwent a pneumonectomy with 65 patients having N2 lymphatic metastasis and 51 patients who accepted neoadjuvant therapy, 51 patients had better 5 -year OS than patients without neoadjuvant therapy (32.7\% vs. $10.7 \%, \mathrm{P}=0.014)$ (19). However, in a subset analysis of study INT0139, Albain (20) found that there is no significant long-term OS benefit from neoadjuvant chemoradiotherapy followed by pneumonectomy compared to definitive chemoradiotherapy in patients with N2 IIIA NSCLC. Of the 164 patients receiving thoracotomy, 10 patients died within 30 days of surgery and most of them died of pneumonectomy. Its impact on OS is thought to be mediated largely through the high post-operative death rate caused by the surgery of pneumonectomy.

However, all of our 100 patients received pneumonectomy, and the 30 -day mortality is only $4.0 \%$, which is less than the 30-day mortality of the study INT0139. Based on this observation, we conclude that patients undergoing pneumonectomy could benefit more from neoadjuvant therapy and achieve a better long-term survival, in contrast to those who did not receive neoadjuvant therapy. In our institute, among those 23 patients with NSCLC and N2 lymphatic metastasis who underwent neoadjuvant therapy, 6 patients $(26.1 \%)$ were observed to be downstaged but had no 5 -year OS benefit than those without lymph node downstage $(\mathrm{P}=0.473)$. However, Paul et al. previously demonstrated that N2 lymph node downstage was an important predictor of prognosis (21). We failed to demonstrate this, but proved the efficacy of neoadjuvant therapy in patients with N2 lymphatic metastasis. This may be attributed to the small sample size of our study.

\section{Conclusions}

Pneumonectomy sometimes is inevitable and necessary in certain type of NSCLC with acceptable perioperative mortality and long-term survival. For patients with NSCLC undergoing pneumonectomy, pathological subtype and nodal stage were independent predictors of OS. When pneumonectomy was inevitable in patients with NSCLC and N2 lymphatic metastasis, a better long-term survival could be achieved among patients receiving neoadjuvant therapy compared to those without neoadjuvant therapy.

\section{Acknowledgments}

Funding: None.

\section{Footnote}

Conflicts of Interest: All authors have completed the ICMJE uniform disclosure form (available at http://dx.doi. org/10.21037/jtd.2020.02.33). The authors have no conflicts of interest to declare.

Etbical Statement: The authors are accountable for all aspects of the work in ensuring that questions related to the accuracy or integrity of any part of the work are appropriately investigated and resolved. This retrospective study was performed under a waiver of authorization approved by the Institutional Review Board of Peking Union Medical College Hospital, Beijing, China (No. S-K1049).

Open Access Statement: This is an Open Access article distributed in accordance with the Creative Commons Attribution-NonCommercial-NoDerivs 4.0 International License (CC BY-NC-ND 4.0), which permits the noncommercial replication and distribution of the article with the strict proviso that no changes or edits are made and the original work is properly cited (including links to both the formal publication through the relevant DOI and the license). See: https://creativecommons.org/licenses/by-nc-nd/4.0/.

\section{References}

1. Torre LA, Bray F, Siegel RL, et al. Global cancer statistics, 2012. CA Cancer J Clin 2015;65:87-108.

2. Graham EA, Singer JJ. Landmark article Oct 28, 1933. Successful removal of an entire lung for carcinoma of the bronchus. By Evarts A. Graham and J. J. Singer. JAMA 1984;251:257-60.

3. Brunelli A, Xiumé F, Refai M, et al. Evaluation of expiratory volume, diffusion capacity, and exercise tolerance following major lung resection: a prospective follow-up analysis. Chest 2007;131:141-7.

4. Smulders SA, Holverda S, Vonk-Noordegraaf A, et al. Cardiac function and position more than 5 years after pneumonectomy. Ann Thorac Surg 2007;83:1986-92.

5. Licker M, Spiliopoulos A, Frey JG, et al. Risk factors for early mortality and major complications following pneumonectomy for non-small cell carcinoma of the lung. Chest 2002;121:1890-7.

6. Shapiro M, Swanson SJ, Wright CD, et al. Predictors of major morbidity and mortality after pneumonectomy 
utilizing the Society for Thoracic Surgeons General Thoracic Surgery Database. Ann Thorac Surg 2010;90:927-34; discussion 934-5.

7. Ludwig C, Stoelben E, Olschewski M, et al. Comparison of morbidity, 30-day mortality, and long-term survival after pneumonectomy and sleeve lobectomy for non-small cell lung carcinoma. Ann Thorac Surg 2005;79:968-73.

8. Qadri SS, Chaudhry MA, Cale A, et al. Short- and longterm outcomes of pneumonectomy in a tertiary center. Asian Cardiovasc Thorac Ann 2016;24:250-6.

9. Tanaka S, Aoki M, Ishikawa H, et al. Pneumonectomy for node-positive non-small cell lung cancer: can it be a treatment option for N2 disease? Gen Thorac Cardiovasc Surg 2014;62:370-5.

10. Krasna MJ, Gamliel Z, Burrows WM, et al. Pneumonectomy for lung cancer after preoperative concurrent chemotherapy and high-dose radiation. Ann Thorac Surg 2010;89:200-6; discussion 206.

11. Weder W, Collaud S, Eberhardt WE, et al. Pneumonectomy is a valuable treatment option after neoadjuvant therapy for stage III non-small-cell lung cancer. J Thorac Cardiovasc Surg 2010;139:1424-30.

12. Broderick SR, Patel AP, Crabtree TD, et al. Pneumonectomy for clinical stage IIIA non-small cell lung cancer: the effect of neoadjuvant therapy. Ann Thorac Surg 2016;101:451-7; discussion 457-8.

13. Hatz RA, Klotz LV. Consequences of pneumonectomy in the early and late phases. Chirurg 2013;84:497-501.

14. Lee H, Ahn YC, Pyo H, et al. Pretreatment clinical mediastinal nodal bulk and extent do not influence survival

Cite this article as: Wang G, Liu L, Zhang J, Li S. The analysis of prognosis factor in patients with non-small cell lung cancer receiving pneumonectomy. J Thorac Dis 2020;12(4):13661373. doi: $10.21037 /$ jtd.2020.02.33 in N2-positive stage IIIA non-small cell lung cancer patients treated with trimodality therapy. Ann Surg Oncol 2014;21:2083-90.

15. Kalathiya RJ, Saha SP. Pneumonectomy for nonsmall cell lung cancer: outcomes analysis. South Med J 2012;105:350-4.

16. Fernandez FG, Force SD, Pickens A, et al. Impact of laterality on early and late survival after pneumonectomy. Ann Thorac Surg 2011;92:244-9.

17. Gu C, Wang R, Pan X, et al. Comprehensive study of prognostic risk factors of patients underwent pneumonectomy. J Cancer 2017;8:2097-103.

18. Marulli G, Verderi E, Zuin A, et al. Outcomes and prognostic factors of non-small-cell lung cancer with lymph node involvement treated with induction treatment and surgical resection. Interact Cardiovasc Thorac Surg 2014;19:256-62; discussion 262.

19. White A, Kucukak S, Bueno R, et al. Pneumonectomy is safe and effective for non-small cell lung cancer following induction therapy. J Thorac Dis 2017;9:4447-53.

20. Albain KS, Swann RS, Rusch VW, et al. Radiotherapy plus chemotherapy with or without surgical resection for stage III non-small-cell lung cancer: a phase III randomised controlled trial. Lancet 2009;374:379-86.

21. Paul S, Mirza F, Port JL, et al. Survival of patients with clinical stage IIIA non-small cell lung cancer after induction therapy: age, mediastinal downstaging, and extent of pulmonary resection as independent predictors. J Thorac Cardiovasc Surg 2011;141:48-58. 